effect of recArg1 regarding an in vivo model called serum transfer arthritis, where we treated C57BL/6J wildtype mice with the recombinant enzyme. Disease severity was then assessed using clinical scores and paw histology.

Results We observed that ARG1 mRNA expression was downregulated from the progression of a precursor to a mature osteoclast. We incubated day 3 osteoclast precursors with recArg1 and observed that addition of $1000 \mathrm{ng} / \mathrm{ml} \mathrm{recArg} 1$ abolished osteoclastogenesis. L-Arginine deprivation led to a decrease in the oxygen consumption rate of osteoclast precursor cells, assessed 48 hour after RANKL addition. Using serum transfer arthritis, an established murine in vivo model, recArg1 treated mice showed reduced disease severity combined with a significant decrease in the presence of osteoclasts. Treatment efficiency was evaluated using an L-Arginine ELISA, where the amino acid was found to be absent in the serum of treated mice.

Conclusions We propose that the amino acid L-Arginine is critical for the development of osteoclasts from myeloid precursors and hypothesise that its abundance, influenced by recArg1 addition, influences development and severity of osteoclast driven diseases.

Disclosure of interest J. Brunner Grant/research support from: Bio Cancer Treatment International Ltd, M. Hofmann Grant/ research support from: Bio Cancer Treatment International Ltd, V. Saferding Grant/research support from: Bio Cancer Treatment International Ltd, A. Vogel Grant/research support from: Bio Cancer Treatment International Ltd, A. Lercher: None declared, P. Cheng Shareholder of: Bio Cancer Treatment International Ltd, G. Schabbauer Grant/research support from: Bio Cancer Treatment International Ltd, S. Blüml Grant/research support from: Bio Cancer Treatment International Ltd

\section{P114 IONISING RADIATION INHIBITS INFLAMMATION IN PATIENTS WITH MUSCULOSKELETAL DISEASES: RADON TREATMENT VS LOW-DOSE RADIATION THERAPY}

${ }^{1} \mathrm{~K}$ Shreder*, 'A Cucu, ${ }^{1} \mathrm{D}$ Kraft, 'S Lehrian, ${ }^{1} \mathrm{~J}$ Kondol, ${ }^{2} \mathrm{G}$ Klein, ${ }^{3} \mathrm{~B}$ Frey, ${ }^{3} \mathrm{U}$ Gaipl, ${ }^{1} \mathrm{C}$ Fournier. 'GSI Helmholtzzentrum für Schwerionenforschung GmbH, Darmstadt; ${ }^{2}$ Association for Spa Research and Medical Practice for Cardiology, Bad Steben; ${ }^{3}$ Department of Radiation Oncology, Universitätsklinikum Erlangen, Erlangen, Germany

\subsection{6/annrheumdis-2018-EWRR2018.129}

Introduction Rheumatoid arthritis (RA) and osteoarthritis (OA) are the most common musculoskeletal diseases (MSD) that affect the joints. Reduced mobility and quality of life are the consequences of the cartilage and bone tissue destruction and the chronic inflammation process, caused by release of bone destruction markers and inflammatory factors including adipokines in the joint. Besides medications, an additional pain relief is achieved by the treatment of patients with low-dose ionising radiation, either as local low-dose radiation therapy (LDRT) or whole-body exposure to radon in radon baths or galleries.

Objectives In the previous work we showed the decrease of serum levels of visfatin and serum carboxy-terminal collagen crosslinks of type-I collagen (CTX-I) in patients treated in radon baths. ${ }^{1}$ In the present study, we analysed serum samples of patients with MSD, who had been treated locally with photon radiation (LDRT). In addition, we analysed differentiation and activity of osteoclasts that were differentiated in vitro from patient-derived monocytes.

Methods Serum samples were collected from patients before and after treatment. Levels of visfatin and CTX-I were measured by ELISA. Monocytes were isolated from blood samples of patients and cultivated with M-CSF and RANKL on bone slices for 2 weeks. Osteoclasts were defined as TRAP and Factin positive cells. TRAP activity was measured in the cell supernatants using TRAP Staining Kit.

Results In the serum of patients treated with LDRT, a trend to reduced concentration of CTX-I was observed directly after the therapy. Further, osteoclasts, differentiated in vitro from LDRT patient-derived monocytes, showed reduced TRAP activity.

Conclusions The observations made in this study so far substantiate that the radiation-induced decrease of CTX-I levels could be one main factor that is related to the attenuation of inflammation and to the decrease of disease activity in the patients with MSD. This hypothesis is endorsed by the observed reduced differentiation and activity of in vitro cultivated patient-derived osteoclasts.

\section{REFERENCE}

1. Cucu Shreder, et al. Front. Immunol 2017;8:882.

Acknowledgements This work was supported by the German Federal Ministry of Education and Research (grant no. 02NUK017A and 02NUK017G, GREWIS), by Landesamt für Gesundheit und Lebensmittelsicherheit Bayern, and by Bayrisches Staatsbad Bad Steben GmbH.

Disclosure of interest None declared

\section{P115 LOW DOSE RADIATION HAS A POSITIVE IMPACT ON BONE METABOLISM IN AN EXPERIMENTAL MODEL OF INFLAMMATORY ARTHRITIS}

'L Deloch*, 'M Rückert, ${ }^{2} \mathrm{AJ}$ Hueber, ${ }^{2} \mathrm{M}$ Herrmann, ${ }^{1} \mathrm{R}$ Fietkau, ${ }^{1} \mathrm{~B}$ Frey, ${ }^{1}$ US Gaipl. ${ }^{1}$ Department of Radiation Oncology; ${ }^{2}$ Internal Medicine 3, Rheumatology and Immunology, Universitätsklinikum Erlangen, Friedrich-Alexander-Universität Erlangen-Nürnberg, Erlangen, Germany

\subsection{6/annrheumdis-2018-EWRR2018.130}

Introduction Rheumatoid arthritis (RA) is, next to inflammation and infiltration of activated immune cells into the synovial joint, characterised by a progressive destruction of cartilage and bone. Although today's treatment options are very effective for many patients, not all of them respond properly or have to reduce medications due to adverse effects. In these patients it is crucial to slow down bone loss and inflammation in a timely manner to prevent further damage. Here, low-dose radiotherapy (LD-RT) could be an option, as it has been shown to ameliorate inflammation and to reduce pain. Using the human $T N F \alpha$ transgenic (hTNF $\alpha$ tg) mouse model as an experimental model of inflammatory arthritis, we revealed that locally applied LD-RT attenuates inflammation in the joints.

Objectives As little is known about the impact of LD-RT on bone metabolism, we thus focused on the effects of LD-RT on bone homeostasis.

Methods Bone marrow-derived osteoclasts (OC) of hTNF $\alpha$ tg mice were differentiated using M-CSF and RANK-L and 
seeded either on plastic or bone slices prior to irradiation to investigate their numbers and function. Osteoblasts (OB) were differentiated from mouse calvariae, irradiated and analysed regarding their differentiation and function using alizarin red stain and $\mathrm{qPCR}$. Hind legs of $\mathrm{hTNF} \alpha$ tg mice were locally irradiated with $0.5 \mathrm{~Gy}$ of X-rays and consecutively analysed using histomorphological methods and $\mu \mathrm{CT}$.

Results Treatment of OC with LD-RT (0.5-2 Gy) lead to decreased differentiation and function, while $\mathrm{OB}$ showed increased mineralization after irradiation with 0.5 Gy. Further, no significant increase of Acp5 and catk at 0.5 Gy was found in OC while OB showed a dose-dependent decrease in RANK$L$ and increase in OPG expression. Also, OPG secretion by $\mathrm{OB}$ was enhanced at $0.5 \mathrm{~Gy}$ and 1 Gy. Histomorphometric analyses showed a decrease of erosive areas and OC numbers in the paws of irradiated animals, a finding that was confirmed in $\mu \mathrm{CT}$ images.

Conclusions We conclude that LD-RT is not only a valuable tool for reduction of inflammation, but also has beneficial effects in restoring a balanced bone homeostasis through increased $\mathrm{OB}$ mineralization and decreased $\mathrm{OC}$ function. In the future, placebo controlled studies are desirable in order to investigate the effects of LD-RT with focus on osteoimmunology in RA in the clinic.

Acknowledgements Supported by the German Federal Ministry of Education and Research (GREWIS, 02NUK017G and GREWIS-alpha, 02NUK050E

Disclosure of interest None declared

\section{P116 HISTONE DEACETYLASE 1: A NOVEL THERAPEUTIC TARGET FOR PATIENTS WITH RHEUMATOID ARTHRITIS}

${ }^{1} \mathrm{~L}$ Göschl, ${ }^{1} \mathrm{~L}$ Müller, ${ }^{1} V$ Saferding, ${ }^{2} \mathrm{~J}$ Bäcklund, ${ }^{3} \mathrm{~S}$ Knapp, ${ }^{4} \mathrm{P}$ Mathias, ${ }^{1} \mathrm{C}$ Scheinecker, ${ }^{5} \mathrm{~W}$ Ellmeier, ${ }^{1} \mathrm{G}$ Steiner, ${ }^{1} \mathrm{M}$ Bonelli*. ${ }^{1}$ Department of Rheumatology, Medical University Vienna, Vienna, Austria; '2Department of Medical Biochemistry and Biophysics, Karolinska Institutet, Stockholm, Sweden; ${ }^{3}$ Research Centre for Molecular Medicine (CeMM), Austrian Academy of Sciences, Vienna, Austria; ${ }^{4}$ Friedrich Miescher Institute for Biomedical Research, Basel, Switzerland; ${ }^{5}$ Division of Immunobiology, Institute of Immunology, Centre for Pathophysiology, Infectiology and Immunology, Medical University of Vienna, Vienna, Austria

\subsection{6/annrheumdis-2018-EWRR2018.131}

Introduction Despite enormous efforts to develop new therapeutic strategies for treatment of rheumatoid arthritis (RA), the large number of non responding patients to currently available drugs underlies the unmet need to identify new therapeutic targets. Certain $\mathrm{CD}^{+} \mathrm{T}$ cells have been shown to be major drivers of inflammation in patients with RA. The expression of their key transcription factors is controlled by histone modifications which includes acetylation of lysine residues mediated by histone deacetylases (HDAC). Indeed, pan HDAC inhibitors have been shown to be a potential therapeutic strategy in the treatment of malignancies. However, major side effects limited the clinical use and underline the need of more specific HDAC inhibitors. Recent data suggest a role of HDAC1 on the migratory capacity and the differentiation of CD $4^{+} \mathrm{T}$ cells in the context of autoimmune encephalitis. ${ }^{1}$

Objectives Our aim was to elucidate the individual role of HDAC1 in a T cell dependent arthritis model, namely the collagen-induced arthritis model (CIA).

Methods Mice with a $\mathrm{T}$ cell specific deletion of HDAC1 (HDAC1 cKO) were generated by using the CD4Cre/LoxP system. At week 8 of age arthritis was induced in wild type
(WT) and HDAC1 cKO mice by immunising with chicken collagen II (CII), emulsified in complete Freund's adjuvant. The animals were scored for clinical signs of arthritis, namely paw swelling and grip strength. Anti-CII antibody levels were determined by ELISA. Various cell subsets, including Th cells, where detected in the blood, the spleen and the draining lymph node by FACS analysis. To test antigen-specific $\mathrm{T}$ cell activation we performed in vitro restimulation of spleen and lymph node cells with collagen II followed by assessment of cytokine production and quantification of the proliferation rate using ${ }^{3} \mathrm{H}$-thymidine incorporation. After 10 weeks mice were sacrificed and paraffin sections of the affected joints were analysed for histomorphologic signs of inflammation, cartilage and bone destruction.

Results Eighty percent of the animals developed serum antiCII anibodies (IgM and $\operatorname{IgG}$ ). No difference in the production of antibody subclasses, especially of pathogenic IgG2c antibodies, were observed between WT and HDAC1 cKO mice. Surprisingly, HDAC1 cKO mice were completely protected and did not develop any clinical signs of arthritis. Accordingly, histological analysis revealed no signs of inflammation, no bone erosion and no appearance of osteoclasts in the joints of HDAC1 cKO mice. In line with our recently published data on the role of HDAC1 on the differentiation and migration of $\mathrm{T}$ cell subsets we suggest that HDAC1 plays a key role in the development of arthritis.

Conclusions Our data show the importance of HDAC1 as a key immune regulator in the pathogenesis of $\mathrm{T}$ cell driven collagen induced arthritis. Therefore it might be considered as an interesting novel therapeutic target in RA.

\section{REFERENCE}

1. Goschl L, et al. A T cell-specific deletion of HDAC1 protects against experimental autoimmune encephalomyelitis. J Autoimmun 2017.

Disclosure of interest None declared

\section{P117 EFFECT OF DIFFERENT JANUS KINASE INHIBITORS ON FIBROBLAST-LIKE SYNOVIOCYTES IN RHEUMATOID ARTHRITIS}

${ }^{1} \mathrm{M}$ Diller*, ${ }^{1} \mathrm{M}$-L Hülser, ${ }^{2} \mathrm{~S}$ Rehart, ${ }^{3,4} \mathrm{M}$ Fleck, ${ }^{1} \mathrm{E}$ Neumann, ${ }^{1} \mathrm{U}$ Müller-Ladner. ${ }^{1}$ Dept. of Rheumatology and Clinical Immunology, Campus Kerckhoff, Justus-Liebig-University Gießen, Gießen; ${ }^{2}$ Dept. of Orthopaedics and Trauma Surgery, Agaplesion Markus Hospital, Frankfurt, ${ }^{3}$ Dept. of Rheumatology and Clinical Immunology, Asklepios Medical Centre Bad Abbach, Bad Abbach; ${ }^{4}$ Dept. of Internal Medicine (I), University Medical Centre Regensburg, Regensburg, Germany

\subsection{6/annrheumdis-2018-EWRR2018.132}

Introduction In 2017, the Janus kinase inhibitors (JAKi) Tofacitinib and Baricitinib were approved for the treatment of Rheumatoid arthritis (RA) in the European Union (EU) by the European Medicines Agency (EMA). Other JAKi like Peficitinib, Filgotinib and Upadacitinib are currently examined in clinical trials. The effect of these substances is mainly based on blocking the IL-6 pathway in immune cells. ${ }^{1}$ However the effect of the different JAKi on activated fibroblast-like synoviocytes from patients with RA (RA-FLS) is not well characterised.

Objectives This study compared the effect of different JAKi on the pro-inflammatory response of activated RA-FLS.

Methods RA-FLS were isolated from synovial tissue of patients with known RA undergoing joint replacement surgery. The 